MATHEMATICS OF COMPUTATION

Volume 77, Number 262, April 2008, Pages 967-983

S 0025-5718(07)01982-5

Article electronically published on September 28, 2007

\title{
RATIONAL GAUSS-CHEBYSHEV QUADRATURE FORMULAS FOR COMPLEX POLES OUTSIDE $[-1,1]$
}

\author{
KARL DECKERS, JORIS VAN DEUN, AND ADHEMAR BULTHEEL
}

\begin{abstract}
In this paper we provide an extension of the Chebyshev orthogonal rational functions with arbitrary real poles outside $[-1,1]$ to arbitrary complex poles outside $[-1,1]$. The zeros of these orthogonal rational functions are not necessarily real anymore. By using the related para-orthogonal functions, however, we obtain an expression for the nodes and weights for rational Gauss-Chebyshev quadrature formulas integrating exactly in spaces of rational functions with arbitrary complex poles outside $[-1,1]$.
\end{abstract}

\section{INTRODUCTION}

Efficient rational Gauss-Chebyshev quadrature formulas were derived in 7 for the case of arbitrary real poles outside the interval $[-1,1]$. Compared to other quadrature rules which are exact for certain classes of rational functions, the effort of computing the nodes and weights is remarkably small, even for extremely high degrees $\left(n>10^{4}\right)$. These formulas were derived in the framework of orthogonal rational functions as described in [1, Chap. 11], but the fact that they are so easy to compute is due to the existence of explicit expressions for Chebyshev rational functions with real poles, which were also derived in [7].

The main purpose of this paper is to extend the results from [7] to the case of arbitrary complex poles outside $[-1,1]$. We will give expressions for the rational functions orthogonal with respect to any of the three Chebyshev weights and discuss the computation of the nodes and weights in the corresponding quadrature formulas. To this end we also study the asymptotic distribution of these nodes, using the theory of logarithmic potentials with external fields. The technique used in [7] to derive most of the results was based on Bernstein-Szegö polynomials, but for the case of complex poles this is no longer possible, since there is no direct connection between the orthogonal rational functions and polynomials orthogonal to a positive varying weight. Also, the nodes in the quadrature formulas are no longer the zeros of the Chebyshev rational functions themselves (these are complex), but are the zeros of so-called para-orthogonal rational functions. It turns out, however, that the resulting formulas are very similar to the ones from [7].

Received by the editor February 9, 2006.

2000 Mathematics Subject Classification. Primary 42C05, 65D32.

Key words and phrases. Quadrature formulas, orthogonal rational functions.

The work of the first two authors was partially supported by the Fund for Scientific Research (FWO), projects 'CORFU: Constructive study of orthogonal functions', grant \#G.0184.02 and, 'RAM: Rational modelling: optimal conditioning and stable algorithms', grant \#G.0423.05, and by the Belgian Programme on Interuniversity Attraction Poles, initiated by the Belgian Federal Science Policy Office. The scientific responsibility rests with the authors.

(C)2007 American Mathematical Society 967

Reverts to public domain 28 years from publication 
In the next section we give the necessary theoretical preliminaries. Sections 34 present the explicit expressions for the orthogonal and para-orthogonal functions and the equations for the nodes and weights. We conclude the article with some numerical examples.

\section{Preliminaries}

The field of complex numbers will be denoted by $\mathbb{C}$ and the Riemann sphere by $\overline{\mathbb{C}}=\mathbb{C} \cup\{\infty\}$. For the real line we use the symbol $\mathbb{R}$ and for the extended real line $\overline{\mathbb{R}}=\mathbb{R} \cup\{\infty\}$. The unit circle and the open unit disc are denoted respectively by

$$
\mathbb{T}=\{z:|z|=1\} \quad \text { and } \quad \mathbb{D}=\{z:|z|<1\} .
$$

We will study orthogonal rational functions (ORF) on the real interval $I=[-1,1]$. The complement of this interval with respect to a set $X$ will be given by $X^{I}$, e.g.

$$
\overline{\mathbb{C}}^{I}=\overline{\mathbb{C}} \backslash I \text {. }
$$

Furthermore, we will use $\mathbb{Z}$ to denote the set of integers. If $b=\lceil a\rceil$ with $a \in \mathbb{R}$, then $b$ is the smallest integer so that $b \geq a$.

Suppose a sequence of poles $A=\left\{\alpha_{1}, \alpha_{2}, \ldots\right\} \subset \overline{\mathbb{C}}^{I}$ is given and define the factors

$$
Z_{k}(x)=\frac{x}{1-x / \alpha_{k}}, \quad k=1,2, \ldots,
$$

and the basis functions

$$
b_{0}=1, \quad b_{k}(x)=b_{k-1}(x) Z_{k}(x), \quad k=1,2, \ldots .
$$

Then the space of rational functions with poles in $A$ is defined as

$$
\mathcal{L}_{n}=\operatorname{span}\left\{b_{0}, \ldots, b_{n}\right\} \text {. }
$$

In the special case of all $\alpha_{k}=\infty$, the expression in (2.1) becomes $Z_{k}(x)=x$ and the expression in (2.2) becomes $b_{k}(x)=x^{k}$. Let $\mathcal{P}_{n}$ denote the space of polynomials of degree less than or equal to $n$ and define

$$
\pi_{n}(x)=\prod_{k=1}^{n}\left(1-x / \alpha_{k}\right)
$$

then we may equivalently write

$$
\mathcal{L}_{n}=\left\{p_{n} / \pi_{n}, p_{n} \in \mathcal{P}_{n}\right\}
$$

We denote the Joukowski Transformation $x=\frac{1}{2}\left(z+z^{-1}\right)$ by $x=J(z)$, mapping the open unit disc $\mathbb{D}$ onto the cut Riemann sphere $\overline{\mathbb{C}}^{I}$ and the unit circle $\mathbb{T}$ onto the interval $I$. The inverse mapping is denoted by $z=J^{i n v}(x)$ and is chosen so that $z \in \mathbb{D}$ if $x \in \overline{\mathbb{C}}^{I}$. With the sequence $A=\left\{\alpha_{1}, \alpha_{2}, \ldots\right\} \subset \overline{\mathbb{C}}^{I}$ we associate a sequence $B=\left\{\beta_{1}, \beta_{2}, \ldots\right\} \subset \mathbb{D}$ so that $\beta_{k}=J^{i n v}\left(\alpha_{k}\right)$.

Given this sequence of complex numbers $B=\left\{\beta_{1}, \beta_{2}, \ldots\right\} \subset \mathbb{D}$, we define the Blaschke factors

$$
\zeta_{k}(z)=\frac{z-\beta_{k}}{1-\bar{\beta}_{k} z}, \quad k=1,2, \ldots,
$$

and the Blaschke products

$$
B_{0}=1, \quad B_{k}(z)=B_{k-1}(z) \zeta_{k}(z), \quad k=1,2, \ldots
$$


With the weight functions

$$
w(x)= \begin{cases}\left(1-x^{2}\right)^{-1 / 2}, & i=1, \\ \left(\frac{1-x}{1+x}\right)^{1 / 2}, & i=2, \\ \left(1-x^{2}\right)^{1 / 2}, & i=3,\end{cases}
$$

we define the inner product of two functions $f(x)$ and $g(x)$ as

$$
\langle f, g\rangle_{w}=\int_{-1}^{1} f(x) \overline{g(x)} w(x) d x .
$$

If $\langle f, f\rangle_{w} \neq 0$ and $\langle g, g\rangle_{w} \neq 0$, then $f(x)$ and $g(x)$ are orthogonal to each other with respect to the weight function $w(x)$ (denoted by $f \perp_{w} g$ ) iff $\langle f, g\rangle_{w}=0$. In the case of $\langle f, f\rangle_{w}=\langle g, g\rangle_{w}=1$, we say that $f(x)$ and $g(x)$ are orthonormal to each other with respect to the weight function $w(x)$. So, assume now that we are given a sequence of arbitrary complex poles $\left\{\alpha_{k}\right\}_{k=1}^{n}$ outside $I=[-1,1]$. Then the rational function $\varphi_{n}(x)$ with poles $\left\{\alpha_{k}\right\}_{k=1}^{n}$ is said to be orthogonal iff $\varphi_{n} \perp_{w} f$ for each $f \in \mathcal{L}_{n-1}$ (also denoted by $\varphi_{n} \perp_{w} \mathcal{L}_{n-1}$ ).

We define the involution operation or substar conjugate of a function $f(z)$ as

$$
f_{*}(z)=\overline{f(\bar{z})}
$$

and the superstar transformation as

$$
f^{*}(z)=\frac{b_{n}(z)}{b_{n *}(z)} f_{*}(z) .
$$

Note that the factor $b_{n}(z) / b_{n *}(z)$ merely replaces the polynomial with zeros $\left\{\bar{\alpha}_{k}\right\}_{k=1}^{n}$ in the denominator of $f_{*}(z)$ by a polynomial with zeros $\left\{\alpha_{k}\right\}_{k=1}^{n}$ so that $\mathcal{L}_{n}^{*}=\mathcal{L}_{n}$.

Furthermore, we define the para-orthogonal rational functions $Q_{n}(x, \tau)$ as

$$
Q_{n}(x, \tau)=\varphi_{n}(x)+\tau \varphi_{n}^{*}(x), \quad \tau \in \mathbb{T}, \quad n \geq 1 .
$$

The use of these para-orthogonal functions lies in the fact that their zeros are simple and real and can be used as nodes in the quadrature formulas. The quadrature formulas follow from the next theorem.

Theorem 2.1. Assume $Q_{n}(x, \tau)=q_{n}(x, \tau) / \pi_{n}(x)$ is regular, i.e. none of the zeros $x_{n k}(\tau)$ of $q_{n}(x, \tau)$ coincides with any of the poles. Define

$$
\lambda_{n k}=\left(\sum_{j=0}^{n-1}\left[\varphi_{j}\left(x_{n k}(\tau)\right) \overline{\varphi_{j}\left(x_{n k}(\tau)\right)}\right]\right)^{-1} .
$$

Then the quadrature formula

$$
\int_{-1}^{1} w(x) f(x) d x \approx \sum_{k=1}^{n} \lambda_{n k} f\left(x_{n k}(\tau)\right)
$$

is exact for $f \in \mathcal{L}_{n-1} \cdot \mathcal{L}_{n-1 *}$. In the special case in which $\alpha_{n}$ is real, this quadrature formula is exact for $f \in \mathcal{L}_{n} \cdot \mathcal{L}_{n-1 *}$ (see [6, p. 490]).

In the case of real poles outside $I$, the expression for the Chebyshev $\operatorname{ORF} \varphi_{n}^{(i)}(x)$ related to the $i^{\text {th }}$ weight in (2.4), as well as expressions for the computation of the nodes and weights in the quadrature formula, are given in the next theorem. For the proof we refer to Theorems 3.4, 4.1 and 4.3 from [7, pp. 313-318]. 
TABLE 1. Definition of $c, d, p$ and $q$ in the function of $i$.

\begin{tabular}{|c|c|c|c|c|}
\hline$i$ & $c$ & $d$ & $p$ & $q$ \\
\hline 1 & 1 & 1 & 1 & -1 \\
\hline 2 & $3 / 2$ & 0 & 1 & 1 \\
\hline 3 & 2 & 0 & 2 & 1 \\
\hline
\end{tabular}

Theorem 2.2. Let $x=J(z) \in \overline{\mathbb{C}}$ and $\alpha_{k}=J\left(\beta_{k}\right) \in \overline{\mathbb{R}}^{I}$. Suppose we define the numbers $c, d, p$ and $q$ for $i=1,2,3$ according to Table 1 , Then the orthonormal rational functions $\varphi_{n}^{(i)}(x)$ with $n \geq 1$ are given by

$$
\varphi_{n}^{(i)}(x)=\sqrt{\frac{2^{i}}{\pi}} \sqrt{1-\beta_{n}^{2}} \frac{q}{2 z^{i-1}+q-3}\left(\frac{z^{i} B_{n-1}(z)}{1-\beta_{n} z}-\frac{q}{\left(z-\beta_{n}\right) B_{n-1}(z)}\right) .
$$

If $n=0$, then $\varphi_{n}^{(i)}(x)$ is given by

$$
\varphi_{0}^{(i)}=\sqrt{\frac{p}{\pi}} .
$$

Furthermore, the nodes for the construction of the rational Gauss-Chebyshev quadrature formulas are the zeros of $\varphi_{n}^{(i)}(x)$. Let $\arctan (y / x)$ refer to the argument of the complex number $x+\mathbf{i} y$ in $[-\pi / 2,3 \pi / 2)$ and define

$$
f_{n}(\theta)=2 \sum_{j=1}^{n-1} \arctan \frac{\sin \theta}{\cos \theta-\beta_{j}}+\arctan \frac{\sin \theta}{\cos \theta-\beta_{n}} .
$$

Let $x_{n k}^{(i)}$ denote the zeros of $\varphi_{n}^{(i)}(x)$ and put $x_{n k}^{(i)}=\cos \theta_{n k}^{(i)}$; then they satisfy the following equation:

$$
f_{n}\left(\theta_{n k}^{(i)}\right)-(n-c) \theta_{n k}^{(i)}=\pi k-d \frac{\pi}{2}, \quad k=1,2, \ldots, n .
$$

Finally, define

$$
g_{n}(x)=2 \sum_{j=1}^{n-1} \frac{\sqrt{1-1 / \alpha_{j}^{2}}}{1-x / \alpha_{j}}+\frac{\sqrt{1-1 / \alpha_{n}^{2}}}{1-x / \alpha_{n}} .
$$

Then the weights in the Gaussian quadrature formulas based on the $\varphi_{n}^{(i)}(x)$ can be given as functions of the nodes $x_{n k}^{(i)}$ as follows:

$$
\lambda_{n k}^{(i)}=2 \pi \frac{1-(1-d)\left[x_{n k}^{(i)}\right]^{i-1}}{i+g_{n}\left(x_{n k}^{(i)}\right)}, \quad k=1,2, \ldots, n .
$$

Note that for all poles equal to infinity, $\varphi_{n}^{(1)}(x)$ becomes the Chebyshev polynomial of the first kind and $\varphi_{n}^{(3)}(x)$ becomes the Chebyshev polynomial of the second kind. Furthermore, we have in this case that $\mathcal{L}_{n}=\mathcal{P}_{n}$ and $\mathcal{L}_{n} \cdot \mathcal{L}_{n-1}=\mathcal{P}_{2 n-1}$. From now on, we will omit the superscript ${ }^{(i)}$ in order not to complicate the notation.

\section{Chebyshev ORF with complex poles}

With the definition of the substar conjugate in section 2, we have that

$$
\zeta_{k *}(z)=\frac{z-\bar{\beta}_{k}}{1-\beta_{k} z}, \quad k=1,2, \ldots,
$$


and consequently that

$$
B_{0 *}=1, \quad B_{k *}(z)=B_{k-1 *}(z) \zeta_{k *}(z), \quad k=1,2, \ldots .
$$

Note that neither the denominator of $\zeta_{k *}$ nor the denominator of $\zeta_{k}^{-1}$ contains a complex conjugate. So with this we can rewrite Lemma 3.1 from [8, pp. 174-175] as follows. The proof is similar to the one of Lemma 3.1 in [8, pp. 174-175], and we omit it.

Lemma 3.1. Suppose $A=\left\{\alpha_{k}\right\}_{k=1}^{n} \subset \overline{\mathbb{C}}^{I}$ and $B=\left\{\beta_{k}\right\}_{k=1}^{n} \subset \mathbb{D}$, and consider the factors $Z_{k}(x), \zeta_{k}(z)$ and $\zeta_{k *}(z)$. Then there exist constants $E$ and $F$ only depending on $A$ and $B$ so that

$$
Z_{k}\left(\frac{z+z^{-1}}{2}\right)=E \zeta_{k *}(z)+F+E \zeta_{k}^{-1}(z)
$$

iff

$$
\alpha_{k}=\left(\beta_{k}+\beta_{k}^{-1}\right) / 2
$$

The explicit forms of these constants $E$ and $F$ are

$$
E=\frac{\left(1+\beta_{k}^{2}\right)^{2}}{2\left(1-\beta_{k}^{2}\right)\left(1-\left|\beta_{k}\right|^{2}\right)} \quad \text { and } \quad F=\frac{\left(\beta_{k}+\bar{\beta}_{k}\right)\left(1+\beta_{k}^{2}\right)}{\left(1-\beta_{k}^{2}\right)\left(1-\left|\beta_{k}\right|^{2}\right)} .
$$

For complex poles outside $I$, it now follows that, if the condition on the placement of the poles in (3.1) is satisfied, then $b_{k}(x)$ is a linear combination of

$$
B_{k}^{-1}(z), B_{k-1}^{-1}(z), \ldots, B_{1}^{-1}(z), B_{0}^{-1}=B_{0 *}, B_{1 *}(z), \ldots, B_{k-1 *}(z), B_{k *}(z) .
$$

We now give explicit expressions for the Chebyshev rational functions with arbitrary complex poles outside $I$. Note the similarity with Theorem 3.4 from [7, p. 313].

Theorem 3.2. Let $x=J(z) \in \overline{\mathbb{C}}$ and $\alpha_{k}=J\left(\beta_{k}\right) \in \overline{\mathbb{C}}^{I}$. Suppose $p$ and $q$ defined as before in Table 1 and let $N$ represent the normalisation factor given by

$$
N=\sqrt{\frac{2^{i}}{\pi}} \sqrt{1-\left|\beta_{n}\right|^{2}}
$$

Then the orthonormal rational functions $\varphi_{n}(x)$ with $n \geq 1$ are given by

$$
\varphi_{n}(x)=\frac{q N}{2 z^{i-1}+q-3}\left(\frac{z^{i} B_{n-1 *}(z)}{1-\beta_{n} z}-\frac{q}{\left(z-\beta_{n}\right) B_{n-1}(z)}\right) .
$$

If $n=0$, the expression in (2.7) still holds.

Before proving Theorem 3.2 we will prove a little lemma that we shall need not only for the proof of Theorem 3.2 but also for some computations in section 4 .

Lemma 3.3. Let $\varphi_{n}(x)$ be given by (3.3). If $x \in \overline{\mathbb{R}}$, then $\overline{\varphi_{n}(x)}$ is given by

$$
\overline{\varphi_{n}(x)}=\frac{q N}{2 z^{i-1}+q-3}\left(\frac{z^{i} B_{n-1}(z)}{1-\bar{\beta}_{n} z}-\frac{q}{\left(z-\bar{\beta}_{n}\right) B_{n-1 *}(z)}\right) .
$$

Proof. In the case of $x \in I$ we have that $|z|=1$ or $\bar{z}=1 / z$ so that $\overline{B_{n *}(z)}=B_{n *}^{-1}(z)$ and $\overline{B_{n}(z)}=B_{n}^{-1}(z)$. Consequently, we have that

$$
\begin{aligned}
\overline{\varphi_{n}(x)} & =\frac{q N z^{i-1}}{2+(q-3) z^{i-1}}\left(\frac{1}{z^{i-1}\left(z-\bar{\beta}_{n}\right) B_{n-1 *}(z)}-\frac{q z B_{n-1}(z)}{1-\bar{\beta}_{n} z}\right) \\
& =\frac{-q^{2} N}{2+(q-3) z^{i-1}}\left(\frac{z^{i} B_{n-1}(z)}{1-\bar{\beta}_{n} z}-\frac{q}{\left(z-\bar{\beta}_{n}\right) B_{n-1 *}(z)}\right) .
\end{aligned}
$$


Furthermore, by filling in the different possibilities for $i$ and $q$, it is easy to see that the equality

$$
\frac{-q^{2}}{2+(q-3) z^{i-1}}=\frac{q}{2 z^{i-1}+q-3}
$$

holds. Finally, note that if $x \in \overline{\mathbb{R}}^{I}$, then $z=\bar{z} \in I$ so that $\overline{B_{n *}(z)}=B_{n}(z)$.

Proof of Theorem 3.2. First, note that with $x=\cos \theta$ and $w(x)=(1-x)^{\mu}(1+x)^{\nu}$, where $\mu$ and $\nu$ belong to $\{ \pm 1 / 2\}$, we get that (see also [2, p. 687] and [8, p. 174])

$$
2 \int_{-1}^{1} w(x) d x=\int_{-\pi}^{\pi} w(\cos \theta)|\sin \theta| d \theta=\int_{-\pi}^{\pi}(1-\cos \theta)^{\mu+\frac{1}{2}}(1+\cos \theta)^{\nu+\frac{1}{2}} d \theta .
$$

Or, with $z=e^{\mathbf{i} \theta}$ and $d \theta=d z / \mathbf{i} z$, this becomes

$$
\begin{aligned}
\int_{-1}^{1} w(x) d x & =\frac{1}{2} \oint\left(\frac{(-1)^{\mu+\frac{1}{2}}}{(2 z)^{\mu+\nu+1}}(z-1)^{2 \mu+1}(z+1)^{2 \nu+1}\right) \frac{d z}{\mathbf{i} z} \\
& =\frac{1}{2} \oint \frac{-q}{2 \mathbf{i}(2 z)^{i}}\left[\frac{q}{2 z^{i-1}+q-3}\right]^{-2} d z,
\end{aligned}
$$

where the integral is over the complex unit circle and the last equality is easily verified by filling in the different possibilities for $i$ and $q$. Second, define

$$
\begin{aligned}
& f_{1}(z)= \frac{-q N^{2}}{2^{i+1} \mathbf{i}}\left(\frac{z^{i} B_{n-1 *}(z) B_{k-1}(z)}{\left(1-\beta_{n} z\right)\left(1-\bar{\beta}_{k} z\right)}\right) \\
& f_{2}(z)= \frac{-q N^{2}}{2^{i+1} \mathbf{i}}\left(\frac{1}{z^{i} B_{k-1 *}(z) B_{n-1}(z)\left(z-\beta_{n}\right)\left(z-\bar{\beta}_{k}\right)}\right) \\
& f_{3}(z)= \frac{N^{2}}{2^{i+1} \mathbf{i}}\left(\frac{B_{n-1 *}(z)}{\left(1-\beta_{n} z\right)\left(z-\bar{\beta}_{k}\right) B_{k-1 *}(z)}\right) \\
& f_{4}(z)= \frac{N^{2}}{2^{i+1} \mathbf{i}}\left(\frac{B_{k-1}(z)}{\left(1-\bar{\beta}_{k} z\right)\left(z-\beta_{n}\right) B_{n-1}(z)}\right) \\
& f_{1}(z)+f_{2}(z)+f_{3}(z)+f_{4}(z) . \text { Then } \\
& \quad\left\langle\varphi_{n}, \varphi_{k}\right\rangle_{w}=\frac{1}{2} \oint f(z) d z .
\end{aligned}
$$

and $f(z)=f_{1}(z)+f_{2}(z)+f_{3}(z)+f_{4}(z)$. Then

Because $f_{1}(z)$ is analytic on $\mathbb{D} \cup \mathbb{T}$, it follows that

$$
\oint f_{1}(z) d z=0 .
$$

Furthermore, it is clear that $f_{2}(z)=f_{1}(\bar{z}) / z^{2}$ so that

$$
\oint f_{2}(z) d z=\oint \frac{f_{1}(\bar{z})}{z^{2}} d z=\oint f_{1}(z) d z=0,
$$

and equivalently

As a result

$$
\oint f_{4}(z) d z=\oint f_{3}(z) d z
$$

$$
\left\langle\varphi_{n}, \varphi_{k}\right\rangle_{w}=\oint f_{3}(z) d z .
$$

If $k \neq n$, then $f_{3}(z)$ is analytic on $\mathbb{D} \cup \mathbb{T}$ as well, so that

$$
\left\langle\varphi_{n}, \varphi_{k}\right\rangle_{w}=0 \text {. }
$$


In the case of $k=n$, however, we obtain

$$
\oint f_{3}(z) d z=\frac{N^{2}}{2^{i+1} \mathbf{i}} \oint \frac{1}{\left(1-\beta_{n} z\right)\left(z-\bar{\beta}_{n}\right)} d z=\frac{N^{2} \pi}{2^{i}\left(1-\left|\beta_{n}\right|^{2}\right)},
$$

where the last equality follows from the residue theorem. So, with $N$ given by (3.2), we get that

$$
\left\langle\varphi_{n}, \varphi_{n}\right\rangle_{w}=1 .
$$

\section{Quadrature Formulas}

For the construction of the rational Gauss-Chebyshev quadrature formulas, the nodes are the zeros of the para-orthogonal function $Q_{n}(x, \tau)$. Before we derive the formulas for the computation of these zeros, note that $\varphi_{n *}(x)=\overline{\varphi_{n}(x)}$ for $x \in \overline{\mathbb{R}}$. Furthermore, we have that

$$
\frac{\zeta_{k *}(z)}{\zeta_{k}(z)}=\left(\frac{\bar{\beta}_{k}^{2}+1}{\beta_{k}^{2}+1}\right) \frac{Z_{k}(x)}{Z_{k *}(x)}, \quad k=1,2, \ldots, n,
$$

so that

$$
\gamma \varphi_{n}^{*}(x)=\frac{B_{n *}(z)}{B_{n}(z)} \overline{\varphi_{n}(x)}, \quad \gamma \in \mathbb{T} .
$$

Define

$$
X(z)=z^{i-1}+\frac{q-3}{2}
$$

then with $\overline{\varphi_{n}(x)}$ given by (3.4) we have that

$$
\begin{aligned}
\gamma \varphi_{n}^{*}(x) & =\frac{q N}{2 X(z)} \frac{B_{n *}(z)}{B_{n}(z)}\left(\frac{z^{i} B_{n-1}(z)}{1-\bar{\beta}_{n} z}-\frac{q}{\left(z-\bar{\beta}_{n}\right) B_{n-1 *}(z)}\right) \\
& =\frac{q N}{2 X(z)} \frac{\zeta_{n *}(z)}{\zeta_{n}(z)}\left(\frac{z^{i} B_{n-1 *}(z)}{1-\bar{\beta}_{n} z}-\frac{q}{\left(z-\bar{\beta}_{n}\right) B_{n-1}(z)}\right) \\
& =\frac{q N}{2 X(z)} \frac{\left(z-\bar{\beta}_{n}\right)\left(1-\bar{\beta}_{n} z\right)}{\left(z-\beta_{n}\right)\left(1-\beta_{n} z\right)}\left(\frac{z^{i} B_{n-1 *}(z)}{1-\bar{\beta}_{n} z}-\frac{q}{\left(z-\bar{\beta}_{n}\right) B_{n-1}(z)}\right) \\
& =\frac{q N}{2 X(z)}\left\{\frac{z^{i} B_{n-1 *}(z)}{1-\beta_{n} z}\left(\frac{z-\bar{\beta}_{n}}{z-\beta_{n}}\right)-\frac{q}{\left(z-\beta_{n}\right) B_{n-1}(z)}\left(\frac{1-\bar{\beta}_{n} z}{1-\beta_{n} z}\right)\right\} .
\end{aligned}
$$

Since $\gamma \in \mathbb{T}$, we may as well absorb it into the constant $\tau$ in the definition of $Q_{n}(x, \tau)$. Assuming that this has been done, we get that

$$
\begin{aligned}
Q_{n}(x, \tau)=\frac{q N}{2 X(z)}\left\{\frac{z^{i} B_{n-1 *}(z)}{1-\beta_{n} z}(\right. & \left.1+\tau \frac{z-\bar{\beta}_{n}}{z-\beta_{n}}\right) \\
& \left.-\frac{q}{\left(z-\beta_{n}\right) B_{n-1}(z)}\left(1+\tau \frac{1-\bar{\beta}_{n} z}{1-\beta_{n} z}\right)\right\} .
\end{aligned}
$$

We can now derive formulas for the computation of the zeros of $Q_{n}(x, \tau)$, as shown in the next theorem.

Theorem 4.1. Let $\arctan (y / x)$ refer to the argument of the complex number $x+\mathbf{i} y$ in $[-\pi / 2,3 \pi / 2)$ and define

$$
\beta_{n, \tau}=\frac{\beta_{n}+\tau \bar{\beta}_{n}}{1+\tau} .
$$


Suppose $c$ and d defined as before in Table 1 and define

$$
\begin{aligned}
f_{n}(\theta)=\sum_{j=1}^{n-1}\left[\arctan \frac{\sin \theta-\Im\left(\beta_{j}\right)}{\cos \theta-\Re\left(\beta_{j}\right)}+\arctan \frac{\sin \theta+\Im\left(\beta_{j}\right)}{\cos \theta-\Re\left(\beta_{j}\right)}\right] & \\
& +\arctan \frac{\sin \theta}{\cos \theta-\beta_{n, \tau}} .
\end{aligned}
$$

Let $x_{n k}(\tau)$ denote the zeros of $Q_{n}(x, \tau)$, put $x_{n k}(\tau)=\cos \theta_{n k}(\tau)$ and put $\tau \in$ $\mathbb{T} \backslash\{-1\}$ in such a way that $\left|\beta_{n, \tau}\right|<1$. Then these zeros satisfy the following equation:

$$
f_{n}\left(\theta_{n k}(\tau)\right)-(n-c) \theta_{n k}(\tau)=\pi k-d \frac{\pi}{2}, \quad k=1,2, \ldots, n .
$$

Proof. The proof is similar to the one of Theorem 4.1 in [7, p. 315]. We only prove the first case $(i=1)$, since the other cases are analogous. First note that $\bar{\beta}_{n, \tau}=\beta_{n, \tau}$ because $\bar{\tau}=1 / \tau$, so that $\beta_{n, \tau}$ is real. From the expression for $Q_{n}(x, \tau)$ we get that the zeros satisfy

$$
z_{n l} B_{n-1 *}\left(z_{n l}\right) B_{n-1}\left(z_{n l}\right)\left(\frac{z_{n l}-\beta_{n, \tau}}{1-\beta_{n, \tau} z_{n l}}\right)=-1=e^{\mathbf{i} \pi(2 l-1)}, \quad l \in \mathbb{Z},
$$

with $z_{n l}=J^{i n v}\left(x_{n l}\right)$. Equation (4.5) has $2 n$ different solutions, which form complex conjugate pairs. Taking the real parts for $l=1, \ldots, n$ we obtain the $n$ zeros of $Q_{n}(x, \tau)$. Note that $\zeta_{n}(z)$ may be written as

$$
\zeta_{n}(z)=\exp \left\{2 \mathbf{i} \arctan \frac{\sin \theta-\Im\left(\beta_{n}\right)}{\cos \theta-\Re\left(\beta_{n}\right)}-\mathbf{i} \theta\right\}
$$

while $\zeta_{n *}(z)$ may be written as

$$
\zeta_{n *}(z)=\exp \left\{2 \mathbf{i} \arctan \frac{\sin \theta+\Im\left(\beta_{n}\right)}{\cos \theta-\Re\left(\beta_{n}\right)}-\mathbf{i} \theta\right\}
$$

and

$$
\frac{z-\beta_{n, \tau}}{1-\beta_{n, \tau} z}=\exp \left\{2 \mathbf{i} \arctan \frac{\sin \theta}{\cos \theta-\beta_{n, \tau}}-\mathbf{i} \theta\right\} .
$$

Some computations complete the proof.

Note that $\tau=-1$ is a critical value for which one zero of $Q_{n}(x, \tau)$ will lie at infinity, and thus outside $I$, which explains the first condition on $\tau$ in Theorem 4.1. If $\tau \neq-1$, then $Q_{n}(x, \tau)$ will have $n$ finite zeros. The second condition on $\tau$, however, is to assure that each zero of $Q_{n}(x, \tau)$ lies within $(-1,1)$, as will be proved in the next theorem.

Theorem 4.2. Each zero of $Q_{n}(x, \tau)$ lies within $(-1,1)$ iff $\tau \neq-1$ and $\tau$ is chosen in such a way that $\left|\beta_{n, \tau}\right|<1$, with $\beta_{n, \tau}$ given by (4.3).

Proof. First note that $Q_{n}(x, \tau)$ always has at least $n-1$ zeros within $(-1,1)$ (see [6. p. 493]). Assuming that $\beta_{n} \in \mathbb{D} \backslash I$, we can deduce from (4.3) that $\left|\beta_{n, \tau}\right|=1$ for

$$
\tau_{1}=-\frac{1-\beta_{n}}{1-\bar{\beta}_{n}}=\cos \xi_{1}+\mathbf{i} \sin \xi_{1} \quad \text { and } \quad \tau_{2}=-\frac{1+\beta_{n}}{1+\bar{\beta}_{n}}=\cos \xi_{2}+\mathbf{i} \sin \xi_{2},
$$

with $\xi_{1}, \xi_{2} \in[-\pi, \pi]$ and $\xi_{1} \neq \xi_{2}$. Filling in these values for $\tau$ in (4.2) results in $Q_{n}\left(1, \tau_{1}\right)=0$ and $Q_{n}\left(-1, \tau_{2}\right)=0$. Now define $\tilde{\xi}_{1}=\min \left\{\xi_{1}, \xi_{2}\right\}$ and $\tilde{\xi}_{2}=$ $\max \left\{\xi_{1}, \xi_{2}\right\}$. Note that the zeros of $Q_{n}(x, \tau)$ move continuously when $\tau$ changes 
continuously and that, if $x_{*}$ is a zero of $Q_{n}\left(x, \tau_{a}\right)$ with $\tau_{a} \neq-1$, then it is a zero of $Q_{n}\left(x, \tau_{b}\right)$ with $\tau_{b} \neq-1$ as well iff $\tau_{b}=\tau_{a}$. So, $\tilde{\xi}_{1}$ and $\tilde{\xi}_{2}$ are limiting values for having exactly $n-1$ zeros of $Q_{n}(x, \tau)$ within $(-1,1)$, and $Q_{n}(x, \tau)$ must have $n$ zeros in $(-1,1)$ on one side of these limiting values. Furthermore, we have that $\tau=-1$ for $\xi= \pm \pi$. As a result, $Q_{n}(x, \tau)$ will have exactly $n-1$ zeros within $(-1,1)$ for $\xi \in\left[-\pi, \tilde{\xi}_{1}\right] \cup\left[\tilde{\xi}_{2}, \pi\right]$ (where $\left|\beta_{n, \tau}\right| \geq 1$ or $\tau=-1$ ) and $n$ zeros within $(-1,1)$ for $\xi \in] \tilde{\xi}_{1}, \tilde{\xi}_{2}\left[\right.$ (where $\left.\left|\beta_{n, \tau}\right|<1\right)$.

If $\beta_{n} \in I$, then $\beta_{n, \tau}=\beta_{n}$, and thus $\left|\beta_{n, \tau}\right|<1$ for every $\tau \neq-1$. If $\tau=-1$, however, we have that $Q_{n}(x, \tau) \equiv 0$ for $x \in \overline{\mathbb{R}}$, and thus $Q_{n}(1,-1)=Q_{n}(-1,-1)=$ 0 as well. Note that

$$
\lim _{\Im\left(\beta_{n}\right) \rightarrow 0} \tau_{1}=\lim _{\Im\left(\beta_{n}\right) \rightarrow 0} \tau_{2}=-1 .
$$

Therefore, we can consider $\beta_{n} \in I$ as a limit case of $\beta_{n} \in \mathbb{D} \backslash I$ with $\Im\left(\beta_{n}\right) \rightarrow 0$ which means that $Q_{n}(x, \tau)$ will have $n$ zeros within $(-1,1)$ for each $\tau \neq-1$.

Note that $\beta_{n, 1}=\Re\left(\beta_{n}\right)$ so that $\left|\beta_{n, 1}\right|<1$ for each $\beta_{n} \in \mathbb{D}$. For every other $\tau \in \mathbb{T}$ it is always possible to find a $\beta_{n} \in \mathbb{D}$ so that $\left|\beta_{n, \tau}\right| \geq 1$. So $\tau=1$ is the best choice for $\tau \in \mathbb{T}$.

Before we derive explicit expressions for the quadrature weights, we study the asymptotic distribution of the nodes $x_{n k}(\tau)$, using some results from logarithmic potential theory. The distribution of the points $x_{n k}(\tau)$ as $n \rightarrow \infty$ depends on the asymptotic distribution of the poles, as shown below. Note that Theorem 2 in 5 ] is a special case of the following theorem, corresponding to the case of real poles outside $I$.

Theorem 4.3. Assume that the sequence of poles $A=\left\{\alpha_{1}, \alpha_{2}, \ldots\right\}$ is bounded away from $I$ and that the asymptotic distribution of the poles is given by a measure $\nu$ on (a subset of) $\overline{\mathbb{C}}^{I}$, i.e. for any continuous function $f$ with compact support,

$$
\lim _{n \rightarrow \infty} \frac{1}{n} \sum_{k=1}^{n} f\left(\alpha_{k}\right)=\int f(z) d \nu(z)
$$

If $\nu=p \delta_{\infty}+(1-p) \nu_{0}$ with $0 \leq p \leq 1$ (where $\delta_{z}$ is the unit measure whose support is the point $z$ ) and

$$
\int \log |t| d \nu_{0}(t)<\infty
$$

then the asymptotic distribution of the zeros of $Q_{n}(x, \tau)$ is given by an absolutely continuous measure $\lambda$ with weight function

$$
\lambda^{\prime}(x)=\frac{1}{\pi} \frac{1}{\sqrt{1-x^{2}}} \int \Re\left\{\frac{\sqrt{t^{2}-1}}{t-x}\right\} d \nu(t)
$$

where the square root is positive for $t>1$ and the branch cut is $[-1,1]$.

Proof. With the notation introduced above, define

$$
\varphi_{n, \tau}(x)=\frac{q N}{2 X(z)}\left(\frac{z^{i} B_{n-1 *}(z)}{1-\beta_{n, \tau} z}-\frac{q}{\left(z-\beta_{n, \tau}\right) B_{n-1}(z)}\right) .
$$


Then obviously the zeros of $\varphi_{n, \tau}(x)$ are exactly the same as those of $Q_{n}(x, \tau)$. Furthermore it is clear that $\varphi_{n, \tau} \perp_{w} \mathrm{E}_{n-1}$. Writing

$$
\varphi_{n, \tau}(x)=\frac{q_{n, \tau}(x)}{\left(1-x / \alpha_{n, \tau}\right) \pi_{n-1}(x)}
$$

this means that

$$
\int_{-1}^{1} q_{n}(x, \tau) p_{n-1}(x) \frac{w(x)}{\left(1-x / \alpha_{n, \tau}\right)\left|\pi_{n-1}(x)\right|^{2}} d x=0
$$

for any $p_{n-1} \in \mathcal{P}_{n-1}$. So $q_{n}$ is an orthogonal polynomial with respect to a varying weight. If we define

$$
f_{n}(x)=\frac{1}{2 n} \log \left[\left(1-x / \alpha_{n, \tau}\right)\left|\pi_{n-1}(x)\right|^{2}\right]
$$

it follows from the weak convergence (4.6) that

$$
\lim _{n \rightarrow \infty} f_{n}(x)=f(x)=\int \log \left|1-\frac{x}{t}\right| d \nu(t) .
$$

According to the theorem on page 124 of [3], the asymptotic zero distribution of $q_{n}$ is then given by a measure $\lambda$ on $I$, which is the unique solution of the integral equation

$$
\int_{-1}^{1} \log \frac{1}{|x-t|} d \lambda(t)+f(x)=C, \quad x \in I,
$$

where $C$ is a constant. The integral in this expression is just the logarithmic potential of the measure $\lambda$. Note that we have

$$
f(x)=-\int \log \frac{1}{|x-t|} d \nu(t)-\int \log |t| d \nu(t) .
$$

The second integral is a finite constant because of equation (4.7) and because the poles are bounded away from $I$ (and thus from zero), and can be absorbed into the constant $C$. The first integral is the logarithmic potential of $\nu$. This shows that $\lambda$ is the so-called "balayage"-measure of $\nu$ onto $I$. For the case of real poles, an explicit expression for this balayage-measure is given on page 122 of [4, but for the more general case, we have to derive it ourselves.

Let $g_{G}(z, a)$ denote the Green function of the domain $G=\overline{\mathbb{C}}^{I}$ with pole at $a$, and $\phi(z)=z+\sqrt{z^{2}-1}$ the conformal map of the domain $G$ onto the exterior of the unit disk with $\sqrt{z^{2}-1}$ positive for real $z>1$ (note that, with our previous definitions, $\left.\phi(z)=1 / J^{i n v}(z)\right)$. Then it follows from [4, p. 122] that

$$
g_{G}(z, a)=-\log \left|\frac{\phi(z)-\phi(a)}{\overline{\phi(a)} \phi(z)-1}\right| .
$$

According to formula (4.42) on page 121, the balayage-measure $\lambda$ is then given by

$$
\lambda^{\prime}(x)=\frac{1}{2 \pi} \int\left(\frac{\partial g_{G}(x, a)}{\partial \mathbf{n}_{+}}+\frac{\partial g_{G}(x, a)}{\partial \mathbf{n}_{-}}\right) d \nu(a)
$$

where $\mathbf{n}_{ \pm}$are the two normals to the interval $(-1,1)$ with $\mathbf{n}_{+}$pointing to the upper half plane and $\mathbf{n}_{-}$to the lower half plane. From $g_{G}(\bar{z}, a)=g_{G}(z, \bar{a})$ it follows that

$$
\frac{\partial g_{G}(x, a)}{\partial \mathbf{n}_{-}}=\frac{\partial g_{G}(x, \bar{a})}{\partial \mathbf{n}_{+}} \text {. }
$$


The definition of the normal derivative reads

$$
\frac{\partial g_{G}(x, a)}{\partial \mathbf{n}_{+}}=\lim _{h \rightarrow 0} \frac{g_{G}(x+\mathbf{i} h, a)-g_{G}(x, a)}{h} .
$$

Using the fact that $x \in I$ and the relations

$$
\begin{aligned}
\phi(x+\mathbf{i} h) & =\phi(x)+\mathbf{i} h \phi^{\prime}(x)+O\left(h^{2}\right), \quad \\
\log |1+h t| & =h \Re\{t\}+O\left(h^{2}\right),
\end{aligned}
$$

and $\phi^{\prime}(x)=\phi(x) / \sqrt{x^{2}-1}$, some computations yield

$$
\frac{\partial g_{G}(x, a)}{\partial \mathbf{n}_{+}}=-\frac{|\phi(a)|^{2}-1}{\sqrt{1-x^{2}}} \Re\left\{\frac{\phi(x)}{[\phi(x)-\phi(a)][\overline{\phi(a)} \phi(x)-1)]}\right\} .
$$

Because of (4.9), replacing $a$ with $\bar{a}$ gives the other normal derivative. Adding both derivatives together, using $\phi^{2}(x)+1=2 x \phi(x)$ and simplifying then gives

$$
\frac{\partial g_{G}(x, a)}{\partial \mathbf{n}_{+}}+\frac{\partial g_{G}(x, a)}{\partial \mathbf{n}_{-}}=\frac{|\phi(a)|^{2}-1}{\sqrt{1-x^{2}}} \cdot \frac{1+|\phi(a)|^{2}-2 x \Re\{\phi(a)\}}{2|\phi(a)|^{2}(x-a)(x-\bar{a})} .
$$

If we put $a=J(b)$, then $\phi(a)=1 / b$, and we may equivalently write

$$
\frac{\partial g_{G}(x, a)}{\partial \mathbf{n}_{+}}+\frac{\partial g_{G}(x, a)}{\partial \mathbf{n}_{-}}=\frac{1-|b|^{2}}{|b|^{2} \sqrt{1-x^{2}}} \cdot \frac{1+|b|^{2}-2 x \Re\{b\}}{2(x-a)(x-\bar{a})} .
$$

A partial fraction expansion of the second factor gives

$$
\frac{1+|b|^{2}-2 x \Re\{b\}}{2(x-a)(x-\bar{a})}=\Re\left\{\frac{1+|b|^{2}-2 a \Re\{b\}}{(x-a)(a-\bar{a})}\right\},
$$

assuming that $a$ is not real. Now use $a-\bar{a}=(b-\bar{b})\left(|b|^{2}-1\right) /\left(2|b|^{2}\right)$ and do some computations to obtain

$$
\frac{\partial g_{G}(x, a)}{\partial \mathbf{n}_{+}}+\frac{\partial g_{G}(x, a)}{\partial \mathbf{n}_{-}}=\frac{1}{\sqrt{1-x^{2}}} \Re\left\{\frac{b-1 / b}{x-a}\right\}=\frac{2}{\sqrt{1-x^{2}}} \Re\left\{\frac{\sqrt{a^{2}-1}}{a-x}\right\} .
$$

This equation is also valid for real $a$ (taking into account the convention for the square root mentioned above); it then reduces to the result from [4, p. 122]. Substituting in (4.8) then proves the theorem.

Once the nodes $x_{n k}(\tau)$ have been computed, the weights can be found. In the following computations, we will omit the $\tau$ in order not to complicate the notation. Let $X(z)$ be given by (4.1) and define

$$
P(z, t)=\frac{1-|t|^{2}}{|z-t|^{2}}
$$

and

$$
a_{j}(z)=\left(1-\left|\beta_{j}\right|^{2}\right)\left\{\frac{z^{2 i} B_{j-1 *}(z) B_{j-1}(z)}{\left(1-\beta_{j} z\right)\left(1-\bar{\beta}_{j} z\right)}+\frac{q^{2}}{\left(z-\beta_{j}\right)\left(z-\bar{\beta}_{j}\right) B_{j-1 *}(z) B_{j-1}(z)}\right\} .
$$

Then we can deduce from (3.3) and (3.4) that for $x \in I$ and $j \geq 1$

$$
2 \pi \varphi_{j}(x) \overline{\varphi_{j}(x)}=\frac{2^{i-1}}{X^{2}(z)} a_{j}(z)-\frac{q(2 z)^{i-1}}{X^{2}(z)}\left(P\left(z, \beta_{j}\right)+P\left(z, \bar{\beta}_{j}\right)\right) .
$$

For $j=0$ we have that $\varphi_{0}=\overline{\varphi_{0}}$ so that

$$
2 \pi \varphi_{0} \overline{\varphi_{0}}=2 p .
$$


So, with $\lambda_{n k}$ given by (2.6), and with

$$
Y_{n}(z)=2 p+\frac{2^{i-1}}{X^{2}(z)} \sum_{j=1}^{n-1} a_{j}(z)
$$

we obtain

$$
\frac{2 \pi}{\lambda_{n k}}=Y_{n}\left(z_{n k}\right)-\frac{q\left(2 z_{n k}\right)^{i-1}}{X^{2}\left(z_{n k}\right)} \sum_{j=1}^{n-1}\left(P\left(z_{n k}, \beta_{j}\right)+P\left(z_{n k}, \bar{\beta}_{j}\right)\right) .
$$

Note that $\left[-q\left(2 z_{n k}\right)^{i-1} / X^{2}\left(z_{n k}\right)\right]^{-1}$ is in fact nothing more than the factor $[1-$ $\left.(1-d)\left(x_{n k}\right)^{i-1}\right]$ we have in Theorem 2.2 and that for $\beta_{k}$ real

$$
P\left(z_{n k}, \beta_{j}\right)=P\left(z_{n k}, \bar{\beta}_{j}\right)=\frac{1-\beta_{j}^{2}}{1-2 \beta_{j} x_{n k}+\beta_{j}^{2}}=\frac{\sqrt{1-1 / \alpha_{j}^{2}}}{1-x_{n k} / \alpha_{j}} .
$$

Hence, if each pole in the sequence is real, then the equality

$$
Y_{n}\left(z_{n k}\right)=-\frac{q\left(2 z_{n k}\right)^{i-1}}{X^{2}\left(z_{n k}\right)}\left[i+P\left(z_{n k}, \beta_{n}\right)\right]
$$

has to be true. Lemma 4.5 shows that equation (4.12) not only holds for every sequence of real poles, but even for every sequence of complex poles with $\beta_{n}$ replaced by $\beta_{n, \tau}$. To prove this, we first need the following lemma.

Lemma 4.4. Let $h_{n, \beta}(z)$ be given by

$$
h_{n, \beta}(z)=\frac{1}{z^{2}-1}\left[z^{i}\left(\frac{1-\beta z}{z-\beta}\right)+\frac{q}{B_{n-1 *}(z) B_{n-1}(z)}\right] ;
$$

then $h_{n, \beta}(z)$ satisfies the following two recursions:

$$
h_{n+1, \beta}(z)=\frac{h_{n, \beta}(z)}{\zeta_{n *}(z) \zeta_{n}(z)}+\frac{\left(1-\left|\beta_{n}\right|^{2}\right) z^{i}}{\left(z-\beta_{n}\right)\left(z-\bar{\beta}_{n}\right)}\left(\frac{1-\beta z}{z-\beta}\right)
$$

and

$$
h_{n+1, \beta}(z)=h_{n, \beta}(z)-\frac{q\left(1-\left|\beta_{n}\right|^{2}\right)}{\left(z-\beta_{n}\right)\left(z-\bar{\beta}_{n}\right) B_{n-1 *}(z) B_{n-1}(z)} .
$$

Proof. The recursions are easily verified by using the definition of $h_{n, \beta}(z)$ and the knowledge that

$$
\left(1-\left|\beta_{n}\right|^{2}\right)\left(z^{2}-1\right)=\left(z-\beta_{n}\right)\left(z-\bar{\beta}_{n}\right)-\left(1-\beta_{n} z\right)\left(1-\bar{\beta}_{n} z\right) .
$$

With this we can now prove the following lemma.

Lemma 4.5. Let $x_{n k}=J\left(z_{n k}\right)$ be the zeros of $Q_{n}(x, \tau)$; then

$$
Y_{n}\left(z_{n k}\right)=-\frac{q\left(2 z_{n k}\right)^{i-1}}{X^{2}\left(z_{n k}\right)}\left[i+P\left(z_{n k}, \beta_{n, \tau}\right)\right] .
$$

Proof. Assume $\beta \in I$ and let $K_{n, \beta}(z)$ and $L_{n, \beta}(z)$ be given by

$$
K_{n, \beta}(z)=\frac{z^{2 i}-q^{2}}{z^{2}-1}+q z^{i-1} P(z, \beta)+\sum_{j=1}^{n-1} a_{j}(z)
$$


and

$$
L_{n, \beta}(z)=h_{n, \beta}(z)\left[z^{i} B_{n-1 *}(z) B_{n-1}(z)\left(\frac{z-\beta}{1-\beta z}\right)-q\right] .
$$

First, we will prove by induction that $K_{n, \beta}(z)=L_{n, \beta}(z)$ if $z \in \mathbb{T}$. For $n=1$ we get that

$$
\begin{aligned}
L_{1, \beta}(z) & =h_{1, \beta}(z)\left[z^{i}\left(\frac{z-\beta}{1-\beta z}\right)-q\right] \\
& =\frac{1}{z^{2}-1}\left[z^{i}\left(\frac{1-\beta z}{z-\beta}\right)+q\right]\left[z^{i}\left(\frac{z-\beta}{1-\beta z}\right)-q\right] \\
& =\frac{z^{2 i}-q^{2}}{z^{2}-1}+\frac{q z^{i}}{z^{2}-1}\left(\frac{z-\beta}{1-\beta z}-\frac{1-\beta z}{z-\beta}\right) \\
& =\frac{z^{2 i}-q^{2}}{z^{2}-1}+q z^{i-1} P(z, \beta)=K_{1, \beta}(z) .
\end{aligned}
$$

Assume now that $K_{n, \beta}(z)=L_{n, \beta}(z)$ for $n \geq 1$. Then we have that

$$
\begin{gathered}
K_{n+1, \beta}(z)=K_{n, \beta}(z)+a_{n}(z)=L_{n, \beta}(z)+a_{n}(z) \\
=h_{n, \beta}(z)\left[z^{i} B_{n-1 *}(z) B_{n-1}(z)\left(\frac{z-\beta}{1-\beta z}\right)-q\right] \\
+\left(1-\left|\beta_{n}\right|^{2}\right)\left[\frac{z^{2 i} B_{n-1 *}(z) B_{n-1}(z)}{\left(1-\beta_{n} z\right)\left(1-\bar{\beta}_{n} z\right)}+\frac{q^{2}}{\left(z-\beta_{n}\right)\left(z-\bar{\beta}_{n}\right) B_{n-1 *}(z) B_{n-1}(z)}\right] \\
=z^{i} B_{n *}(z) B_{n}(z)\left(\frac{z-\beta}{1-\beta z}\right)\left[\frac{h_{n, \beta}(z)}{\zeta_{n *}(z) \zeta_{n}(z)}+\frac{\left(1-\left|\beta_{n}\right|^{2}\right) z^{i}}{\left(z-\beta_{n}\right)\left(z-\bar{\beta}_{n}\right)}\left(\frac{1-\beta z}{z-\beta}\right)\right] \\
-q\left[h_{n, \beta}(z)-\frac{q\left(1-\left|\beta_{n}\right|^{2}\right)}{\left(z-\beta_{n}\right)\left(z-\bar{\beta}_{n}\right) B_{n-1 *}(z) B_{n-1}(z)}\right] .
\end{gathered}
$$

From the recursions (4.13) and (4.14) in Lemma 4.4, it follows that

$$
K_{n+1, \beta}(z)=h_{n+1, \beta}(z)\left[z^{i} B_{n *}(z) B_{n}(z)\left(\frac{z-\beta}{1-\beta z}\right)-q\right]=L_{n+1, \beta}(z) .
$$

Second, note that

$$
\frac{z^{2 i}-q^{2}}{z^{2}-1}=2^{2-i} p X^{2}(z)+i q z^{i-1}
$$

so that

$$
K_{n, \beta}(z)=2^{2-i} p X^{2}(z)+i q z^{i-1}+q z^{i-1} P(z, \beta)+\sum_{j=1}^{n-1} a_{j}(z) .
$$

Finally, we have that $K_{n, \beta_{n, \tau}}\left(z_{n k}\right)=L_{n, \beta_{n, \tau}}\left(z_{n k}\right)=0$ so that

$$
2^{2-i} p X^{2}\left(z_{n k}\right)+\sum_{j=1}^{n-1} a_{j}\left(z_{n k}\right)=-q z_{n k}^{i-1}\left[i+P\left(z_{n k}, \beta_{n, \tau}\right)\right] .
$$

The equality in (4.15) now follows by using the definition of $Y_{n}(z)$ given by (4.10). 
In the case of complex poles, equation (4.11) does not hold. Instead with

$$
R(x, \beta)=1-2 \Re(\beta) x+|\beta|^{2} \quad \text { and } \quad I(x, \beta)=4[\Im(\beta)]^{2}\left(1-x^{2}\right)
$$

we have that

$$
P(z, \beta)+P(z, \bar{\beta})=\frac{2 R(x, \beta)\left(1-|\beta|^{2}\right)}{R^{2}(x, \beta)-I(x, \beta)} .
$$

Finally, the weights can now be computed using the following theorem.

Theorem 4.6. Let $R(x, \beta)$ and $I(x, \beta)$ be given by (4.16) and define

$$
g_{n}(x)=2 \sum_{j=1}^{n-1} \frac{R\left(x, \beta_{j}\right)\left(1-\left|\beta_{j}\right|^{2}\right)}{R^{2}\left(x, \beta_{j}\right)-I\left(x, \beta_{j}\right)}+\frac{1-\beta_{n, \tau}^{2}}{1-2 \beta_{n, \tau} x+\beta_{n, \tau}^{2}} .
$$

Then the weights in the Gaussian quadrature formulas based on the para-orthogonal function $Q_{n}(x, \tau)$ can be given as functions of the nodes $x_{n k}(\tau)$ as follows:

$$
\lambda_{n k}=2 \pi \frac{1-(1-d)\left[x_{n k}(\tau)\right]^{i-1}}{i+g_{n}\left(x_{n k}(\tau)\right)}, \quad k=1,2, \ldots, n .
$$

\section{NUMERICAL EXAMPLES}

In this section we only give some examples of the use of these quadrature formulas. Unlike in the case for real poles [7, the numerical considerations for the fast and efficient construction of these formulas are rather involved. The main ideas are of course the same as in 7, such as using the asymptotic zero distribution to obtain initial values for Newton's method etc., but a description of a complete software implementation (covering both the real and complex case) and a detailed numerical analysis will be given elsewhere.

Let $\tau$ be fixed to one, and assume the sequences of poles $A_{n}=\left\{\alpha_{1}, \ldots, \alpha_{n-1}, \alpha_{n}\right\}$, $B_{n}=\left\{\alpha_{1}, \ldots, \alpha_{n-1}, \infty\right\}$ and $C_{n+1}=\left\{\alpha_{1}, \ldots, \alpha_{n-1}, \infty, \infty\right\}$ with $\alpha_{k} \in \overline{\mathbb{C}}^{I}$ fixed in advance. We will consider integrals of the form

$$
I_{3}\left(f_{j}\right)=\int_{-1}^{1} \sqrt{1-x^{2}} f_{j}(x) d x, \quad j=1,2, \ldots,
$$

and their approximation by

$$
I_{3, n}\left(f_{j}\right)=\sum_{k=1}^{n} \lambda_{n k}^{(3)} f_{j}\left(x_{n k}^{(3)}\right)
$$

using sequence $A_{n}$. Note that $I_{3}\left(f_{j}\right)$ can be written as

$$
I_{3}\left(f_{j}\right)=\int_{-1}^{1} \sqrt{\frac{1-x}{1+x}}(1+x) f_{j}(x) d x=\int_{-1}^{1} \sqrt{\frac{1-x}{1+x}} g_{j}(x) d x=I_{2}\left(g_{j}\right),
$$

which can be approximated by

$$
I_{2, n}\left(g_{j}\right)=\sum_{k=1}^{n} \lambda_{n k}^{(2)} g_{j}\left(x_{n k}^{(2)}\right)
$$

using the sequence $B_{n}$. Furthermore, $I_{3}\left(f_{j}\right)$ can also be written as

$$
I_{3}\left(f_{j}\right)=\int_{-1}^{1} \frac{1-x^{2}}{\sqrt{1-x^{2}}} f_{j}(x) d x=\int_{-1}^{1} \frac{h_{j}(x)}{\sqrt{1-x^{2}}} d x=I_{1}\left(h_{j}\right),
$$


TABLE 2. Relative error on the approximation of $I_{3}\left(f_{1, n}\right)$ by $I_{3, n}\left(f_{1, n}\right), I_{2, n}\left(g_{1, n}\right)$ and $I_{1, n+1}\left(h_{1, n}\right)$ for several values of $n$.

\begin{tabular}{|c|ccc|ccc|}
\hline$\omega$ & \multicolumn{3}{|c|}{$3+2 \mathbf{i}$} & \multicolumn{3}{c|}{$-0.5+0.05 \mathbf{i}$} \\
\hline$n$ & $\Delta_{3, r}\left(f_{1, n}\right)$ & $\Delta_{2, r}\left(g_{1, n}\right)$ & $\Delta_{1, r}\left(h_{1, n}\right)$ & $\Delta_{3, r}\left(f_{1, n}\right)$ & $\Delta_{2, r}\left(g_{1, n}\right)$ & $\Delta_{1, r}\left(h_{1, n}\right)$ \\
\hline 3 & $2.2 \mathrm{e}-16$ & $2.2 \mathrm{e}-16$ & $3.3 \mathrm{e}-16$ & $2.7 \mathrm{e}-14$ & $9.4 \mathrm{e}-15$ & $1.6 \mathrm{e}-14$ \\
\hline 5 & $5.0 \mathrm{e}-16$ & $1.7 \mathrm{e}-16$ & $3.3 \mathrm{e}-16$ & $6.6 \mathrm{e}-14$ & $3.4 \mathrm{e}-14$ & $5.3 \mathrm{e}-14$ \\
\hline 9 & $3.2 \mathrm{e}-16$ & $6.4 \mathrm{e}-16$ & $8.0 \mathrm{e}-16$ & $8.8 \mathrm{e}-14$ & $9.1 \mathrm{e}-14$ & $9.6 \mathrm{e}-14$ \\
\hline 17 & $1.4 \mathrm{e}-15$ & $1.6 \mathrm{e}-15$ & $1.4 \mathrm{e}-15$ & $2.2 \mathrm{e}-13$ & $1.6 \mathrm{e}-13$ & $2.0 \mathrm{e}-13$ \\
\hline 33 & $4.1 \mathrm{e}-15$ & $3.5 \mathrm{e}-15$ & $3.8 \mathrm{e}-15$ & $3.5 \mathrm{e}-13$ & $3.4 \mathrm{e}-13$ & $3.8 \mathrm{e}-13$ \\
\hline
\end{tabular}

which can be approximated by

$$
I_{1, n+1}\left(h_{j}\right)=\sum_{k=1}^{n+1} \lambda_{n+1, k}^{(1)} h_{j}\left(x_{n+1, k}^{(1)}\right)
$$

using the sequence $C_{n+1}$. From Theorem 2.1 it follows that each of these approximations is exact if $f_{j} \in \mathcal{L}_{n-1} \cdot \mathcal{L}_{n-1 *}$. For each example that follows, define the relative error $\Delta_{i, r}(f)$ by

$$
\Delta_{i, r}(f)=\left|\frac{I_{i}(f)-I_{i, n}(f)}{I_{i}(f)}\right| .
$$

The exact solutions $I_{i}(f)$ were calculated in multiprecision with Maple 9.5. The other computations were done in double precision using Matlab 7.

Example 5.1. The first function $f_{1, n}(x)$ to be considered is given by

$$
f_{1, n}(x)=\frac{1}{\left(x^{2}-2 \Re(\omega) x+|\omega|^{2}\right)^{(n-1) / 2}}, \quad \omega \in \overline{\mathbb{C}}^{I},
$$

for $n$ odd, which has poles of order $(n-1) / 2$ in $\omega$ and $\bar{\omega}$. So let

$$
\alpha_{k}= \begin{cases}\omega & k=\text { odd }, \\ \bar{\omega} & k=\text { even }, \quad k=1, \ldots, n,\end{cases}
$$

with $\omega=3+2 \mathbf{i}$ respectively $\omega=-0.5+0.05 \mathbf{i}$. Then Table 2 gives the relative error for several values of $n$. This function should be integrated exactly and the relative error is indeed of the order of machine precision.

Example 5.2. The second function $f_{2}(x)$ to be considered is taken from [9, p. 169]. In this case we have

$$
f_{2}(x)=\frac{\pi x / \omega}{\sinh (\pi x / \omega)}, \quad \omega \in \overline{\mathbb{R}}^{I},
$$

which has simple poles at the integer multiples of $\mathbf{i} \omega$. So let

$$
\alpha_{k}=(-1)^{k}\lceil k / 2\rceil \mathbf{i} \omega, \quad k=1, \ldots, n,
$$

for $n$ odd, with $\omega=1.1$ respectively $\omega=1.001$. Then Table 3 gives the relative error for several values of $n$. 
TABLE 3. Relative error on the approximation of $I_{3}\left(f_{2}\right)$ by $I_{3, n}\left(f_{2}\right), I_{2, n}\left(g_{2}\right)$ and $I_{1, n+1}\left(h_{2}\right)$ for several values of $n$.

\begin{tabular}{|c|ccc|ccc|}
\hline$\omega$ & \multicolumn{3}{|c|}{1.1} & \multicolumn{3}{c|}{1.001} \\
\hline$n$ & $\Delta_{3, r}\left(f_{1}\right)$ & $\Delta_{2, r}\left(g_{1}\right)$ & $\Delta_{1, r}\left(h_{1}\right)$ & $\Delta_{3, r}\left(f_{1}\right)$ & $\Delta_{2, r}\left(g_{1}\right)$ & $\Delta_{1, r}\left(h_{1}\right)$ \\
\hline 3 & $7.3 \mathrm{e}-4$ & $4.2 \mathrm{e}-7$ & $7.3 \mathrm{e}-4$ & $1.1 \mathrm{e}-3$ & $9.7 \mathrm{e}-7$ & $1.1 \mathrm{e}-3$ \\
\hline 5 & $2.1 \mathrm{e}-7$ & $2.1 \mathrm{e}-14$ & $2.1 \mathrm{e}-7$ & $4.5 \mathrm{e}-7$ & $9.0 \mathrm{e}-14$ & $4.5 \mathrm{e}-7$ \\
\hline 9 & $1.8 \mathrm{e}-16$ & 0 & $1.8 \mathrm{e}-16$ & $1.9 \mathrm{e}-16$ & $1.9 \mathrm{e}-16$ & $5.8 \mathrm{e}-16$ \\
\hline 17 & 0 & 0 & 0 & $1.9 \mathrm{e}-16$ & $3.8 \mathrm{e}-16$ & $3.8 \mathrm{e}-16$ \\
\hline 33 & $1.8 \mathrm{e}-16$ & $3.7 \mathrm{e}-16$ & $1.8 \mathrm{e}-16$ & $1.9 \mathrm{e}-16$ & $1.9 \mathrm{e}-16$ & $1.9 \mathrm{e}-16$ \\
\hline
\end{tabular}

TABLE 4. Relative error on the approximation of $I_{3}\left(f_{3}\right)$ by $I_{3, n}\left(f_{3}\right), I_{2, n}\left(g_{3}\right)$ and $I_{1, n+1}\left(h_{3}\right)$ for several values of $n$.

\begin{tabular}{|c|ccc|}
\hline$\omega$ & \multicolumn{3}{|c|}{0.05} \\
\hline$n$ & $\Delta_{3, r}\left(f_{3}\right)$ & $\Delta_{2, r}\left(g_{3}\right)$ & $\Delta_{1, r}\left(h_{3}\right)$ \\
\hline 101 & $3.9 \mathrm{e}-1$ & $1.3 \mathrm{e}-2$ & $3.8 \mathrm{e}-1$ \\
\hline 201 & $2.9 \mathrm{e}-2$ & $8.2 \mathrm{e}-16$ & $2.9 \mathrm{e}-2$ \\
\hline 401 & $4.5 \mathrm{e}-14$ & $4.9 \mathrm{e}-15$ & $3.7 \mathrm{e}-14$ \\
\hline 801 & $1.1 \mathrm{e}-14$ & $1.4 \mathrm{e}-14$ & $7.8 \mathrm{e}-15$ \\
\hline 1601 & $6.3 \mathrm{e}-15$ & $2.7 \mathrm{e}-15$ & $1.6 \mathrm{e}-14$ \\
\hline 3201 & $5.5 \mathrm{e}-15$ & $9.4 \mathrm{e}-15$ & $4.9 \mathrm{e}-15$ \\
\hline
\end{tabular}

Example 5.3. The last function $f_{3}(x)$ to be considered is analogous to the one in Example 5.4 from [7. In this case we have

$$
f_{3}(x)=\sin \left(\frac{1}{x^{2}+\omega^{2}}\right), \quad \omega \in \overline{\mathbb{R}} \backslash\{0\} .
$$

This function has an essential singularity in $x=\mathbf{i} \omega$ and $x=-\mathbf{i} \omega$. For $\omega>0$ but very close to 0 , this function is extremely oscillatory near these singularities. Since an essential singularity can be viewed as a pole of infinity multiplicity, this suggests taking

$$
\alpha_{k}=\left\{\begin{array}{ll}
\mathbf{i} \omega & k=\text { odd }, \\
-\mathbf{i} \omega & k=\text { even },
\end{array} \quad k=1, \ldots, n,\right.
$$

for $n$ odd. Table 4 gives the relative error for several values of $n$, with $\omega=0.05$.

\section{Conclusion}

We have presented an extension of the expression for the Chebyshev orthogonal rational functions with arbitrary real poles outside $[-1,1]$ to arbitrary complex poles outside $[-1,1]$. The zeros of these orthogonal rational functions are not necessarily real anymore. By using the related para-orthogonal functions, however, we obtained an expression for the nodes and weights for rational Gauss-Chebyshev quadrature formulas integrating exactly in spaces of rational functions with arbitrary complex poles outside $[-1,1]$. 


\section{REFERENCES}

[1] A. Bultheel, P. González-Vera, E. Hendriksen, and O. Njåstad. Orthogonal Rational Functions, volume 5 of Cambridge Monographs on Applied and Computational Mathematics. Cambridge University Press, 1999. MR.1676258 (2000c:33001)

[2] L. Daruis, P. González-Vera, and O. Njåstad. Szegő quadrature formulas for certain Jacobitype weight functions. Math. Comp., 71:683-701, 2001. MR.1885621 (2002k:41043)

[3] A. A. Gonchar and E. A. Rakhmanov. Equilibrium measure and the distribution of zeros of extremal polynomials. Math. USSR Sbornik, 53:119-130, 1986.

[4] E. B. Saff and V. Totik. Logarithmic potentials with external fields, volume 316 of Grundlehren der mathematischen Wissenschaften. Springer, Berlin Heidelberg, 1997. MR 1485778 (99h:31001)

[5] W. Van Assche and I. Vanherwegen. Quadrature formulas based on rational interpolation. Math. Comp., 61(204):765-783, 1993. MR1195424(94a:65014)

[6] J. Van Deun and A. Bultheel. Orthogonal rational functions and quadrature on an interval. $J$. Comput. Appl. Math., 153(1-2):487-495, 2003. MR:1985717(2004e:42043)

[7] J. Van Deun, A. Bultheel, and P. González Vera. On computing rational Gauss-Chebyshev quadrature formulas. Math. Comp., 75:307-326, 2006. MR2176401 (2006e:41060)

[8] P. Van gucht and A. Bultheel. A relation between orthogonal rational functions on the unit circle and the interval $[-1,1]$. Comm. Anal. Th. Continued Fractions, 8:170-182, 2000. MR $1789681(2001 \mathrm{~h}: 42037)$

[9] J.A.C. Weideman and D.P. Laurie. Quadrature rules based on partial fraction expansions. Numerical Algorithms, 24:159-178, 2000. MR1784997(2001f:65032)

Department of Computer Science, K. U. Leuven, B-3001 Heverlee, Belgium

E-mail address: karl.deckers@cs.kuleuven.be

Department of Computer Science, K. U. Leuven, B-3001 Heverlee, Belgium

Current address: Department of Mathematics and Computer Science, Universiteit Antwerpen, B-2020 Antwerpen, Belgium

E-mail address: joris.vandeun@ua.ac.be

Department of Computer Science, K. U. Leuven, B-3001 Heverlee, Belgium

E-mail address: adhemar.bultheel@cs.kuleuven.be 